\title{
Mass spectrometry for the characterization and quantification of engineered inorganic nanoparticles
}

\author{
Jose M. Costa-Fernández *, Mario Menéndez-Miranda ${ }^{1}$, Diego Bouzas-Ramos ${ }^{1}$, \\ Jorge Ruiz Encinar, Alfredo Sanz-Medel ${ }^{* *}$ \\ Department of Physical and Analytical Chemistry, Faculty of Chemistry, University of Oviedo, Julian Claveria 8, 33006 Oviedo, Spain
}

\section{A R T I C L E I N F O}

\section{Keywords:}

Mass spectrometry

Engineered nanoparticles

Hybrid techniques

Nanoanalysis

Characterization

ICP-MS

MALDI

Electrospray ionization

Field-flow fractionation

\begin{abstract}
A B S T R A C T
Advancements in nanoscience enabled the synthesis of a diverse array of engineered nanomaterials. The precise control of the composition and quality of such nanomaterials is required to ensure their eventual successful application. Also importantly, the potentially adverse environmental and/or human health effects resulting from exposure to nanoparticles (NPs) is still a critical but underexplored research area. To understand and assess the potentially harmful effects of NPs a proper knowledge of their physicochemical properties is required.

Among the different available analytical tools for the characterization and quantification of engineered inorganic NPs, mass spectrometry (MS) offers outstanding capabilities. The analytical capabilities of different MS-based techniques, including elemental and molecular detection, and hybrid tools derived from their coupling with different separation approaches, to identify, characterize and quantify NPs is here revised. A forward look into trends on the use of MS for more complete chemical analysis of engineered inorganic NPs is finally attempted.
\end{abstract}

(C) 2016 Elsevier B.V. All rights reserved.

\section{Contents}

1. Introduction

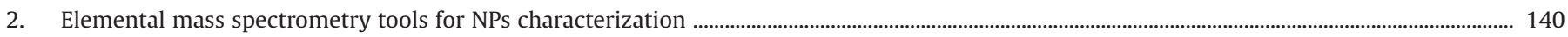

2.1. Direct quantification and elemental characterization of engineered NPs ................................................................................................. 140

2.2. Determination of NPs in biodistribution and toxicological studies via ICP-MS ............................................................................................ 141

2.3. Single particle-ICP-MS methodologies ................................................................................................................................................................. 141

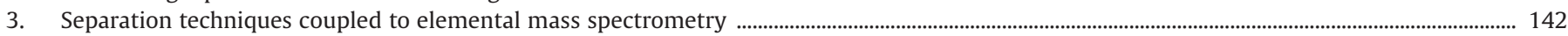

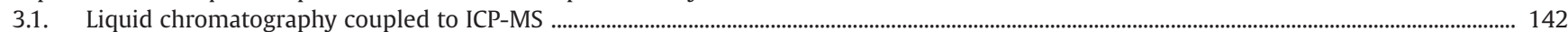

3.2. Asymmetric flow field-flow fractionation coupled to ICP-MS ……............................................................................................................ 143

3.3. Ion mobility analysis coupled with ICP-MS ..................................................................................................................................................... 143

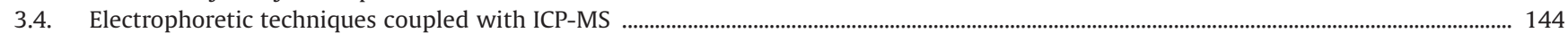

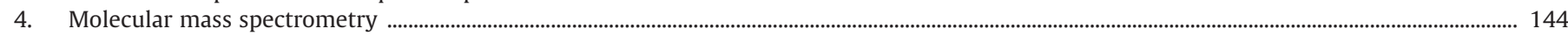

4.1. Matrix-assisted laser desorption/ionization .................................................................................................................................................... 144

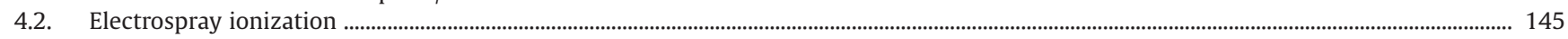

5. Conclusions and outlook on the future of mass spectrometry for engineered nanoparticles ................................................................................... 145

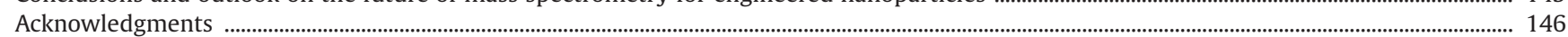

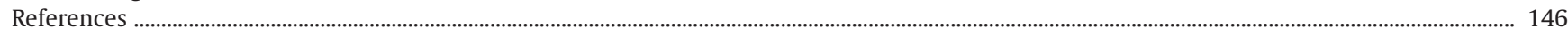

\footnotetext{
* Corresponding author. Tel.: +34 985102970; fax: +34-985103125.

E-mail address: jcostafe@uniovi.es (J.M. Costa-Fernández).

** Corresponding author. Tel.: +34 985103484; fax: +34-985103125.

E-mail address: asm@uniovi.es (A. Sanz-Medel).

1 These authors contributed equally to this work.
}

\section{Introduction}

Research at the nanometre scale, aiming at creating novel nanomaterials with better properties that those of conventional materials, has undergone a rapid growth in recent years. In particular, nanoparticles (NPs) are defined as nanomaterials having all three dimensions ( $\mathrm{x}, \mathrm{y}$ and $\mathrm{z}$ ) in the nanoscale [1]. To understand and 
exploit the high potential of NPs, a knowledge of their morphological and chemical properties, and of the final quality of the nanoproducts (e.g. homogeneity, stability, etc.) is required. On the other hand, the widespread use of NPs in many different areas, including industry, energy, electronics, medicine, food, bioanalysis, etc., is increasing the risks that such nanomaterials enter eventually into our environment and in the human body. However, the harmful effects (toxicity risks) of the increasing number of NPs entering the environment still remain broadly unknown. The assessment of NPs fate and their potential risk to the environment are very involved. In fact, new detection modes and determinations of mass and number of the NPs present in the environment, along with a comprehensive physicochemical characterization of such NPs [2], are urgently demanded. That is, nowadays there is an urgent need of improved analytical tools able to detect, control and characterize the synthesised NPs, not only to improve their synthesis and final desired properties but also to enable us a better understanding of NPs behaviour, final fate and the potential risks to human health that their release may pose.

It must be considered that there is a dual relationship between NPs and analytical chemistry, based on the role played by the corresponding nanomaterial. Actually, nanoparticles considered as analytes require novel analytical tools to achieve their complete characterization and determination. Alternatively, NPs can be considered as tools to improve the performance of existing analytical methods. This article will focus mainly on the more general basic side, that is, the use of mass spectrometry (MS) as an appropriate analytical tool to characterize and determine engineered nanoparticles [3].

MS is gaining growing importance in this context of NPs characterization and quantitation at extremely low levels (e.g. $\mathrm{ng} \mathrm{L}^{-1}$ ) $[4,5]$. Analytical techniques based on mass spectrometry could provide both elemental and molecular information of the desired NPs. The compatibility of MS techniques with virtually any type of sample, the MS extremely high sensitivity and its easy on-line coupling with separation techniques (to obtain real-time speciation information) are promoting most valuable and novel insights into the nature of NPs, on their potential uses and their actual applications.

Here we present an overview on recent advances and evolution trends of mass spectrometric techniques both to control their synthesis and to achieve a complete final characterization of engineered inorganic NPs.

In this vein, the application of elemental-MS techniques will be described first: in fact, NPs chemical composition, core/surface ligand stoichiometries and even NP size and number concentrations can be determined by such technique. Next, the use of hyphenated approaches by resorting to a previous separation step, to characterize polydispersed samples will be discussed. In this context, NPs analytical applications not only require methods capable of detecting NPs at relevant concentrations (sometimes at the trace level) but also the assessment of the sought functionalization of the NPs for a given application (e.g. efficiency of bioconjugation of target biomolecules to NPs). The revision would not be complete without a look into molecular mass spectrometry increasing role for NPs characterization. The outstanding capabilities of modern molecular mass spectrometry in analytical chemistry are rendering MS-based techniques more and more indispensable to obtain deeper information about the NPs, its derivatives and the dynamics of reactions using such probes in bioscience. A forward look into the near future envisaged for MS-based techniques for NPs characterization is also included.

\section{Elemental mass spectrometry tools for NPs characterization}

As many engineered NPs have a core made of metallic elements, NP detection and quantification in complex matrices (e.g. in nanobiotechnology or in environmental and toxicological studies) can be advantageously achieved by resorting to accurate elemental quantification of NPs cores. Taking advantage of the high number of atoms present in the core of a single NP, and the exceptional features of inductively coupled plasma-mass spectrometry (ICP-MS) for high sensitive and robust elemental detections (detection limits down to $\mathrm{pg} \mathrm{g}^{-1}$ with a wide dynamic range up to 9 orders of magnitude), one of the most widespread detector nowadays for the identification, quantification and elemental characterization of NPs is ICP-MS. Additionally, ICP-MS virtual matrix independence, along with plasma-based efficient vaporization, atomization and ionization of most elements (multi-element and multi-isotope analysis) explains why ICP-MS is increasingly used to provide information about NPs size, size distribution, mass and number NPs concentrations, detailed composition or the presence of low levels of impurities in NPs and its derivatives. Some illustrative examples of the impact of such measurements are described below.

\subsection{Direct quantification and elemental characterization of engineered NPs}

The direct elemental analysis of samples containing NPs usually requires acid digestion for sample preparation. However, when possible, the direct introduction of NPs slurries into the ICP constitutes an advantageous alternative that avoids the always tedious and sometimes complex sample dissolution needed before analysis. In this vein, an ICP-MS method for the quantitative determination of Au NPs suspended in aqueous solutions, with sizes ranging from 5 to $20 \mathrm{~nm}$ has been reported [6]. The obtained results showed no significant differences compared to the values of this determination (same Au NPs) after digestion. For NP colloidal dispersion introduction matrix-tolerant nebulizers or electrothermal vaporization (ETV) can be used. ETV-ICP-MS offers a robust, particle size independent, method for quantifying metal(loid oxide) NPs, such as Ag, $\mathrm{Al}, \mathrm{Sb}_{2} \mathrm{O}_{3}, \mathrm{SnO}_{2}, \mathrm{TiO}_{2}$ and $\mathrm{ZnO} \mathrm{NPs}$ and their agglomerates in environmental samples [7]. It allows a multi-elemental determination of NPs using comparatively low sample volumes.

However, most NPs samples usually require acid digestion (e.g. nitric acid was used for Ag NPs digestion, while aqua regia was successfully employed for Au and Pt NPs and CdSe/ZnS Quantum Dots dissolution) in a microwave oven before ICP-MS analysis.

Other NPs made of metal oxides cores, which are highly resistant to such strongly oxidizing acids, might need some additional reagents. For instance, some methods have been developed to determine $\mathrm{TiO}_{2}$ NPs, after digestion with an oxidizing mixture containing hydrofluoric acid (i.e. in food, personal care products, wastewater and biosolids) [8].

Most analytical applications, require that, after the synthesis of the NPs and derivatives, the final product should be isolated, as pure as possible. However, in some cases up to $15 \%$ residual free metal was found in purified samples. Therefore, a full characterization of the NPs and of any concomitant inorganic contaminant are highly important. In this way, ICP-MS enabled a rapid and simultaneous determination of multi-elemental trace impurities in high-purity $\mathrm{NPs}$ of $\mathrm{TiO}_{2}, \mathrm{CeO}_{2}, \mathrm{ZnO}$ and $\mathrm{SiO}_{2}$, after microwave-assisted acid digestion [9].

ICP-MS has been employed as well in combination with UV-vis spectroscopy, transmission electron microscopy (TEM) and X-ray diffraction (XRD), in the elemental characterization of colloidal dispersion of PbSe [10], CdTeSe and CdTeS nanocrystals [11]. The ICP-MS results showed an intriguing nonstoichiometric $\mathrm{Pb}$ to Se ratio, leading to a faceted spherical nanocrystal model composed of a quasi-stoichiometric core terminated by a $\mathrm{Pb}$ surface shell. Isotope dilution-ICP-MS analysis allowed the quantification of the elemental content and distribution of Cd and Se in QDs, made of a core of CdSe and a shell of $\mathrm{ZnS}$, all along the different steps of the 

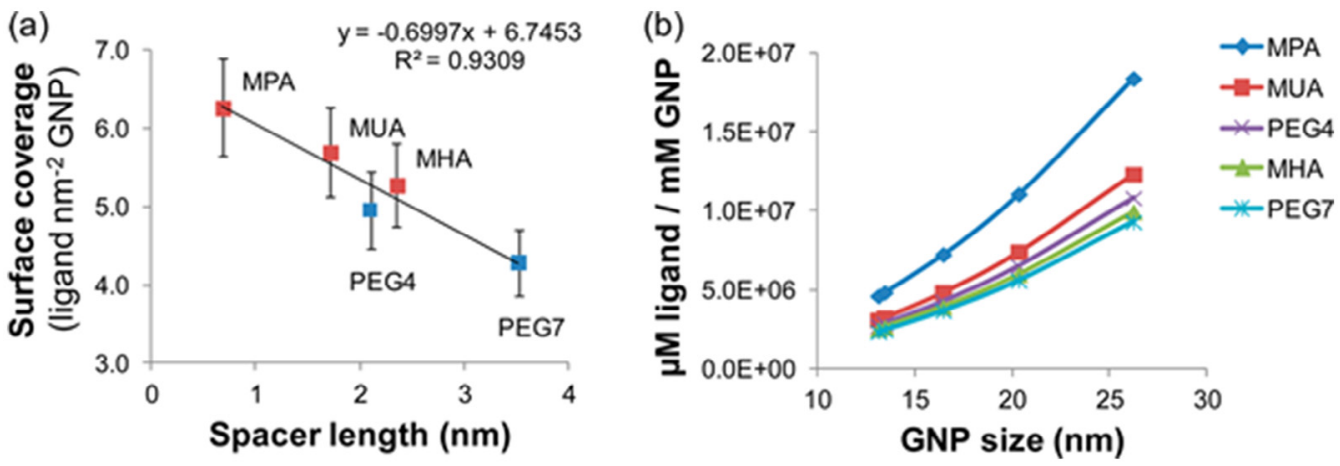

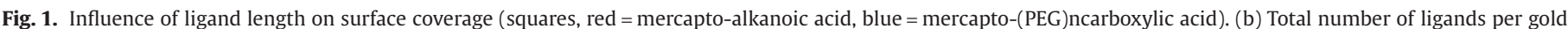

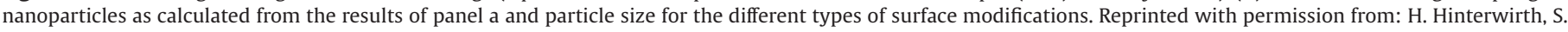

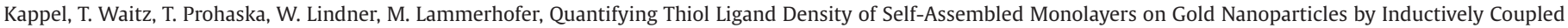
Plasma-Mass Spectrometry, ACS Nano 7 (2013) 1129-1136. Copyright (2013) American Chemical Society.

synthesis. Again, the results indicated the nonstoichiometric nature of the CdSe core and the formation of a CdS/ZnS mixed-shell in the QDs. Besides, an accurate determination of the number of atoms of Cd and Se per synthesized QD was reported [12].

For most applications using NPs, it is imperative to determine "nanoparticle number concentration" in terms of the number of NPs in a given volume of the sample [3]. Such value could be precisely determined combining information from ICP-MS with other conventional techniques used in nanoanalysis. As an example, recently DHLA-capped HgSe NPs were synthesized and characterized using a pool of analytical tools. In this approach, the determination of nanoparticle number concentration relies on the simultaneous quantification of $\mathrm{Hg}$ and Se contained in the NPs core by ICP-MS [13], in combination with the information from XRD and TEM measurements.

ICP-MS analysis not only enables a reliable elemental characterization of engineered NPs, demonstrated in the above examples, but it also provides a valuable information, in derivatives and bioconjugates, in order to establish a relationship between the metal content (from the core of the nanoparticle) and the $S$ content (from the biomolecule or ligand used for surface-modification of the NPs). This molar ratio (core metal/S) can be very accurately measurde by modern ICP-MS systems, and then used to estimate the core/ligand stoichiometry in functionalized NPs. In this sense, simultaneous ICP-MS measurement of mass concentrations of Au and $S$ in digested solutions of $L$-cysteine-modified Au NPs has been reported to determine the NP coverage densities of cysteines [14] and the final quantification of the ligand density of the surface coverage has been calculated. This density turned out to be dependent on the ligand length (see Fig. 1), and on the NP diameter [15]. Most recently, a modern triple quadrupole ICP-MS in combination with different complementary analytical techniques, has been reported for the precise determination of the NP number concentration and the ligand density of engineered water-soluble HgSe NPs capped with dihydrolipoic acid ligands [13].

\subsection{Determination of NPs in biodistribution and toxicological studies via ICP-MS}

During the past few years, many studies have been reported on inorganic engineered NPs monitoring via ICP-MS to establish the biodistribution patterns (mapping) of the NPs in living organisms.

An adequate toxicological study requires evaluation of the interaction of the NP with a given organism (from the very absorption until the final elimination of the NPs). In this sense, reliable information of the biological distribution of the NPs in the scrutinized organism can be obtained just by ICP-MS monitoring these inorganic- based engineered NPs (even at extremely low concentration levels). For instance, the biodistribution of Au NPs with different sizes (15, 50,100 and $200 \mathrm{~nm}$ ) has been studied in mice after intravenous administration of a sodium alginate solution of such NPs [16]. The ICPMS results revealed that the tissue distribution of the Au NPs was size-dependent and most of the Au was demonstrated to be accumulated in liver and spleen. Similarly, ICP-MS measurements enabled evaluation of the distribution and interaction of Ag NPs with cells in the brain [17] and to study the biological behavior of CdSeS QDs in vivo [18].

Moreover, inorganic NPs can also degrade themselves after interacting with cells or tissues by release of metal ions, causing toxic effects to the biological organism. In this vein, ICP-MS has been used to quantify the release of $\mathrm{Ag}(\mathrm{I})$ ions from $\mathrm{Ag} \mathrm{NPs}$, a parameter of great value to shed light about the cytotoxicity effects of these $\mathrm{Ag}$ NPs on their uptake by cells [19].

Acid decomposition (using a microwave oven) of tissues, organs, plasma or fractions of red blood cells for functionalized NPs core determinations has been recently reported [20]. Besides, the determination of $\mathrm{TiO}_{2} \mathrm{NPs}$, of Au nanoshells or of casein NPs carrying cisplatin by ICP-MS have been already reviewed [21], studying NPs kinetics and preferred accumulation in mice body. Also, the pharmacokinetics and the prolonged accumulation and elimination of the popular CdTe/ZnS QDs in mice was explored [20].

\subsection{Single particle-ICP-MS methodologies}

The technique of single particle ICP-MS (sp-ICP-MS) was developed by Degueldre and Favarger [22] more than a decade ago for the detection of model (rutile, alumina and goethite) and natural (montmorillonite) colloids. sp-ICP-MS is based on introducing a discrete flow of a NP suspension sufficiently diluted (NP number concentration below $10^{8} \mathrm{~L}^{-1}$ ) into the plasma in order to detect just one NP per reading, where every single NP produces a pack of ions which results in a transient elemental signal (of less than $0.5 \mathrm{~ms}$ duration). Such resolved transient signal recorded from the ion cloud generated by a single NP in the plasma can be measured by using fast data acquisition frequencies $\left(10^{4}-10^{5} \mathrm{~Hz}\right)$ [23]. On the other hand, by using low acquisition frequencies $(100-1000 \mathrm{~Hz})$, although the ion cloud is recorded as a pulse, it is possible to determine number concentrations and the elemental mass contents per NP. Furthermore, the core size and the number size distributions of the NPs can be determined, provided that some additional information about the shape, composition and density of the NPs is available. Here, it is worth noting that about $18 \mathrm{~nm}$ is the attainable size detection limit that can be detected for metal NPs by sp-ICP-MS [23]. On 

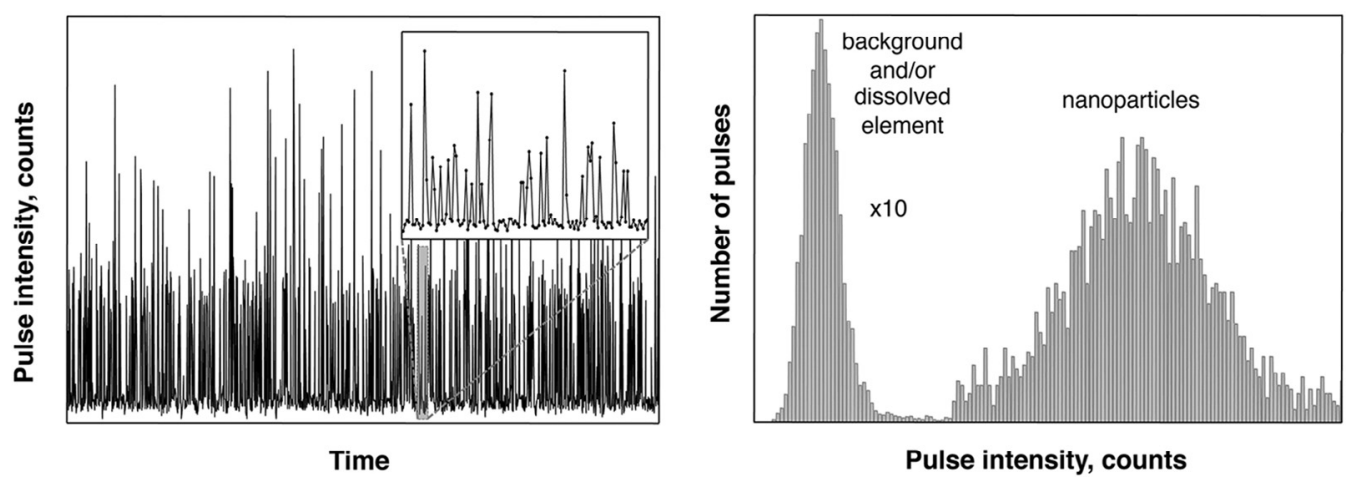

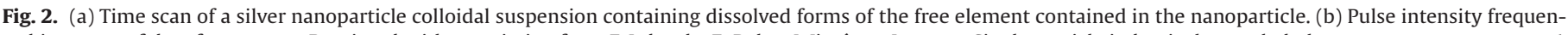

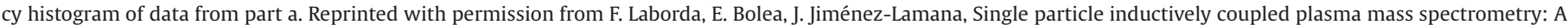
powerful tool for nanoanalysis, Anal. Chem. 86 (2014) 2270-2278. Copyright (2014) American Chemical Society.

the other hand, in practice, NP number concentration limits of detection in the range of $10^{4}-10^{6} \mathrm{~L}^{-1}$ have been reported [24].

The analytical potential of sp-ICP-MS has been already demonstrated for the detection and quantification of Ag NPs in environmental samples (e.g. wastewaters, effluent from treatment plants, etc.) and living organisms [23]. Ag NPs and dissolved $\mathrm{Ag}(\mathrm{I})$ were differentially quantified in water samples (a typical time scan and the signal distribution histogram of a Ag nanoparticle suspension containing dissolved forms of the element are shown in Fig. 2) [24]. Besides, the feasibility of size characterization, assessment of NPs mass percent, their isotopic abundance and stability for $\mathrm{Ag}$ nanowires, $\mathrm{TiO}_{2}, \mathrm{ZnO}$ and $\mathrm{CeO}_{2} \mathrm{NPs}$ by sp-ICP-MS have been also reported [25]. Most recently, a critical review reports the detection and characterization of a wide variety of NPs ( $\mathrm{Au}, \mathrm{Ag}, \mathrm{TiO}_{2}$ and $\mathrm{SiO}_{2}$, among others) in complex matrices, including wastewaters, blood, foods and biological tissues [26]. sp-ICP-MS has also been applied to assess the release of NPs from plastic food containers and to investigate the fate of different metal NPs in several in vitro studies (e.g. study of tissues of organism exposed previously to the NPs) [26].

Although quadrupole instruments provide NP size detection limits of around $20 \mathrm{~nm}$, a microdroplet generator installed in a time-offlight or a sector-field mass analyzer have enabled measuring metal NPs with around $10 \mathrm{~nm}$ size detection limit [27]. The improvement of the few types of commercially available ICP-MS instruments is mandatory in order to get better information about NPs chemical composition, number and mass concentrations, multi-element capabilities for heterogeneous engineered NPs, size and sizedistributions of the NPs at mass concentration levels down to ng $\mathrm{L}^{-1}$ $[23,28]$. This technology could play a major role in the implementation of NPs eventual safety regulations [29].

\section{Separation techniques coupled to elemental mass spectrometry}

A key parameter in nanotechnology is the production of NPs with controlled and well-characterized sizes [30]. Additionally, size distribution should be monitored to clarify whether free or aggregated NPs are in the samples both, in cellular internalization efficiency and in cytotoxicity studies. Therefore a special attention has been paid to the development of analytical methodologies able to provide accurate information in terms of NPs size distribution [26], being dynamic light scattering (DLS) and TEM two of the most popular and efficient approaches for such purposes.

For appropriate size characterization, isolation of the nanomaterials in their native dispersed state is typically needed. Techniques for size distribution analysis of NPs in polydispersed samples include different separation techniques coupled to different detectors, including UV-visible spectroscopy, light scattering and elemental mass spectrometry. Particularly, ICP-MS may provide invaluable information about elemental stoichiometries within the NPs as well as to obtain quantitative elemental information to control the different processes (e.g. recoveries after any chromatographic separation).

\subsection{Liquid chromatography coupled to ICP-MS}

Liquid chromatography (particularly size-exclusion chromatography, SEC) may offer reliable and quantitative information about shape and nanocrystal size distributions in complex polydispersed samples. Such information provided by SEC separations, added to its low cost, fast operation and reliability, make SEC strategies an attractive option for standardizing nanomaterials directly from the colloidal dispersion [31-33].

After those first developments, SEC coupled to ICP-MS has been successfully applied to the size-characterization of many different NPs in several varied matrices [34,35].

One important limitation found with SEC separation for characterization of CdSe quantum dots (QDs) and their bioconjugation to antibodies was reported by Trapiella et al. [34]. The authors observed that, depending on the used experimental conditions, a strong degradation of the polymer coating of the QDs could take place during the SEC separation (affecting the column recovery and the quality of the characterization). It should be noted that such polymer degradation can be also relevant when NPs are applied in practical biological applications (e.g. partial NP polymer coating degradation has been observed when NPs were incubated in cell cultures) [36].

This adsorption interaction observed between the NPs and the SEC stationary phase [34,37], is responsible for its gradual replacement by hydrodynamic chromatography (HDC). HDC has turned out to be the most promising liquid chromatographic technique for this porpoise, in terms of the higher recoveries, shorter analysis times, and good resolution range (typically from 5-300 nm) [38]. The application of HDC is increasing since 2009 [38], when the effectiveness of such separations combined with different detectors (e.g. ICPMS) was demonstrated for NPs size and concentration determination in different matrices. Since then, different authors have employed HDC coupled to mass spectrometry detection for the characterization of NPs. For instance, HDC coupled online to sp-ICP-MS enabled the simultaneous determination of NP size, NP number concentration and metal content in solutions containing inorganic NPs [39]. The analytical potential of such approach was demonstrated for the analysis of drinking water samples spiked with AuNPs reference 


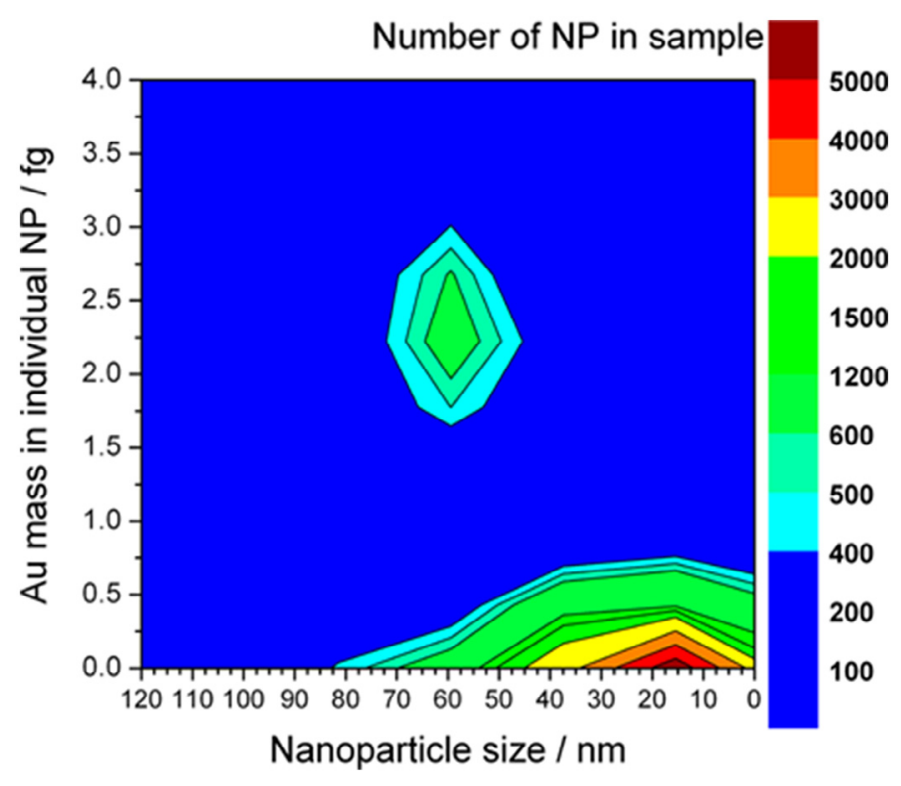

Fig. 3. Plot showing the analysis of a drinking water sample spiked with AuNPs reference materials having NPs nominal sizes of 60 and $30 \mathrm{~nm}$, at concentrations of 100 and $50 \mathrm{ng} \mathrm{Au} \mathrm{L}{ }^{-1}$, respectively. Reprinted with permission from: F. Laborda, E. Bolea, J. Jiménez-Lamana, Single particle inductively coupled plasma mass spectrometry: A powerful tool for nanoanalysis, Anal. Chem. 2012, 84, 6454-6462. Copyright (2012) American Chemical Society.

materials having different NPs nominal sizes at concentrations at the ng Au L $\mathrm{L}^{-1}$ level (see Fig. 3), and reported limits of detection for $60 \mathrm{~nm}$ Au NPs of approximately $2.2 \mathrm{ng} \mathrm{Au} \mathrm{L}^{-1}$ (expressed in terms of NP number concentrations, 600 AuNPs $\mathrm{mL}^{-1}$ ). The strong features of such hyphenated approach for NP characterization are: a) extremely high element sensitivity, b) good selectivity, c) reliable NP size and d) NPs number concentration information.

In a similar way, Roman et al. [40] has demonstrated the potential of the coupling HDC with sp-ICP-MS for the simultaneous determination of dissolved Ag and AgNPs in human plasma and blood. In a single analysis the authors were able to determine the concentration of ionic Ag, the size distribution of AgNPs, and the total number and mass concentrations of the NPs.

\subsection{Asymmetric flow field-flow fractionation coupled to ICP-MS}

One of the main limitations of liquid chromatography to separate NPs in colloidal dispersion is the limited separation range of the technique. Additionally, problems of degradation or aggregation effects of the NPs often take place during the chromatographic separation, derived from undesirable interactions between the NPs and the used solid stationary phase (thus, significantly affecting the reliability of the NPs characterization carried out).

As an alternative to conventional liquid chromatographic techniques, asymmetric flow field-flow fractionation techniques (AF4) have emerged as one of the preferred separation tools for the characterization and analysis of NPs (mainly due to the high separative resolution power at the required sizes range and the absence of the stationary phase that potentially could modify the analyte). AF4 is a flow-assisted technique suited to separate nano-sized particles by their hydrodynamic diameter [41]. Perhaps one of the more serious limiting factors of AF4 for NPs analysis is the sometimes low sample recoveries, (due to undesirable but likely analyte adsorptions in the separation membrane). However, high efforts have been directed during the last years to overcome such drawback [42].

So far, AF4 techniques have been used to characterize the synthesis and growth of different NPs (such as water-soluble Au and
QDs) [43] and to detect $\mathrm{TiO}_{2}$-NPs in commercial products (e.g. sunscreen lotion) [44] using varied detectors. The on-line coupling of AF4 with ICP-MS for NPs characterization has been also described, allowing the development of a rather flexible method to characterize the size, polydispersity, and metal concentrations as a function of the NPs diameter [45].

Separation and identification of NPs of different composition, but with similar particle diameter, coexisting in heterogeneous suspensions have been thoroughly assessed by the AF4 separation coupled on-line to molecular (fluorescence) and elemental (ICPMS) detectors [46]. In this recent work we observed that after simple application of typical bulk molecular techniques, the information obtained from the composition of the sample (colloidal dispersion of QDs synthesized in the laboratory) was far away from the real situation. Fortunately, the AF4 separation coupled to a dual detection ICP-MS/fluorescence provided differential fractograms which proved the presence of a mixture of different size/nature metal NPs coexisting in the different colloidal dispersions under study. Following that approach, the authors were able very recently to separate the excess of free QDs from the QDs bioconjugated to antibodies in their mixtures. Such separations coupled on line to ICP-MS, enabled not only to demonstrate the chemical composition of different NPs in the mixtures, but also the precise determination of the efficiency of the bioconjugations under different reaction conditions (see Fig. 4) [47].

Interestingly, the use of ICP-MS as elemental detector after the AF4 separation also provides useful isotopic information [48]. This makes isotope dilution analysis (IDA) another choice to achieve a more accurate quantification of NPs. In this context, J. Gigault et al. [49] developed a new methodology for isotopically enriched silver nanoparticle $\left({ }^{109} \mathrm{Ag}_{\text {enr }} \mathrm{NP}\right)$ detection and characterization. Authors showed [49] that AF4-ICP-MS combination makes it possible to determine the mass isotopic signature of ${ }^{109} \mathrm{Ag}_{\mathrm{enr}} \mathrm{NPs}$ as a function of their size and optical properties. This feature assures the necessary specificity for tracing and differentiating labeled ${ }^{109} \mathrm{Ag}$ enr NPs (e.g. naturally occurring) from anthropogenic analogs, and so it opens the door to address modern relevant scientific challenges concerning the transport and fate of nanomaterials in natural systems.

As mentioned before, perhaps one of the most active trends in elemental mass spectrometry for the analysis and characterization of NPs is the use of sp-ICP-MS, described before, and the coupling AF4-ICP-MS. Recently, the performance of both approaches was compared for silver NPs [50]. While AF4-ICP-MS showed better sensitivity than sp-ICP-MS in terms of size (DLs of $2 \mathrm{~nm}$ vs $20 \mathrm{~nm}$, respectively), sp-ICP-MS offers better sensitivity in terms of large enough NPs concentrations (e.g. for a $60 \mathrm{~nm}$ nanoparticle size, DLs obtained by sp-ICP-MS was of only $2.2 \mathrm{ng} \mathrm{L}^{-1}$ ).

\subsection{Ion mobility analysis coupled with ICP-MS}

Ion mobility spectrometry (IMS) allow gaseous ions separation on the basis of their size/charge ratios as well as their interactions with a buffer gas. This separation coupled with MS detectors, has been widely employed to tackle structural and size problems in volatile and nonvolatile compounds [51]. Now, such hybrid technique is also starting to be exploited for NPs characterization [52,53].

In a pioneering work, the use of nanoelectrospray IMS combined with off-line ICP-MS for determining NPs in aqueous solution demonstrated excellent capabilities for size and NPs metal content determinations. The resolving power exhibited by IMS along with the high elemental sensitivity offered by ICP-MS are the main features that make this combination attractive for NPs analysis [52]. Coupling of differential mobility to ICP-MS was evaluated for the analysis of gold NPs. The approach enabled NP gas-phase size separation, provided information about number- and mass-based NPs 


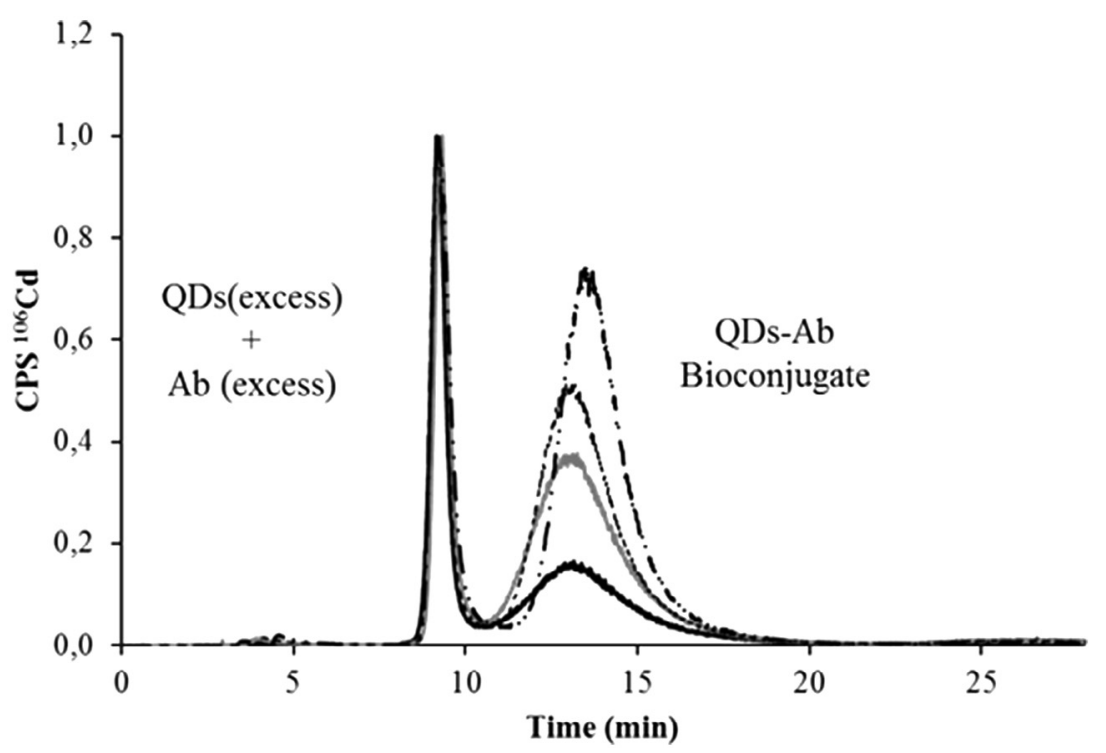

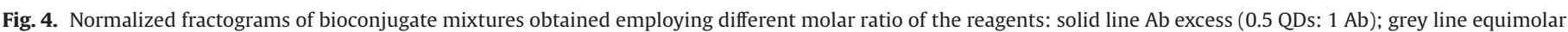

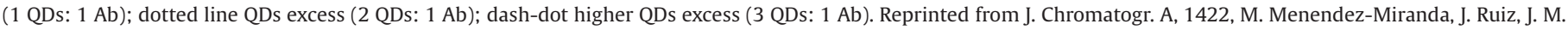

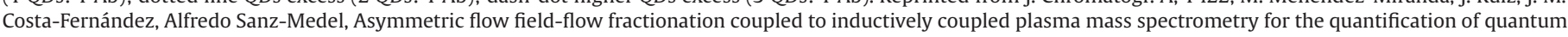
dots bioconjugation efficiency, 247-252, Copyright (2015), with permission from Elsevier.

distributions and allowed the determination of the elemental compositions and the number of atoms per nanoparticle [53].

\subsection{Electrophoretic techniques coupled with ICP-MS}

Electrophoretic techniques, based on the migration of charged species in a liquid under the influence of an applied electric field, have been also applied to characterize and determine NPs [54]. The coupling of capillary electrophoresis (CE) to elemental detection techniques such as ICP-MS has been already described for metal speciation purposes [55]. However, size and concentration of metallic NPs determinations by CE-ICP-MS have only recently been introduced [56,57].

CE separation coupled on-line to ICP-MS was proposed for the identification and accurate size characterization of NPs in complex media [56]. Through mass scanning and Gaussian fitting of the observed electropherogram peaks, the authors obtained multidimensional information on chemical compositions, size distributions, and ionic species of multiple NPs in a single run. In the same idea, CE-ICP-MS was used for rapid and high resolution speciation and characterization of metallic (e.g. gold, platinum, and palladium) NPs in a dietary supplement [57]. The method was successfully applied to the analysis of commercially available metallic nanoparticle-based dietary supplements, and a good agreement between the particle sizes evaluated by CE-ICP-MS and transmission electron microscopy techniques was reported.

\section{Molecular mass spectrometry}

NPs surface must be coated with appropriate molecules to confer NPs the desirable stability and/or to provide the surface with a specific functionality. The design of robust and versatile surface chemistries for NPs passivation has been investigated during the last years, aiming at:

a) achieve NPs solubilization and stabilization in aqueous media or biological buffers, while keeping their original photophysical and size properties. b) provide adequate reactive groups in the NPs surface for further bioconjugations to other biomolecules [58].

An appropriate control of the NP surface coating will facilitate the efficiency of such processes. Particularly, when NPs are designed to be used in bioanalytical applications, they are commonly conjugated to biological probes, including nucleic acids, proteins, antibodies or small molecules. Again, control of the efficiency of those functionalization reactions is mandatory to ensure an optimal analytical application of the sought bioconjugates.

Perhaps one of the most challenging analytical steps in characterizing such functionalized NPs is the determination of the stoichiometries, if any, and molecular formulas. In this sense, molecular MS techniques could provide such key information. Some of the more recent and analytically relevant examples of using molecular MS (MALDI- and ESI-MS approaches) for effective functionalized NPs characterization are discussed below.

\subsection{Matrix-assisted laser desorption/ionization}

Matrix-assisted laser desorption/ionization mass spectrometry (MALDI-MS) has become an extremely popular and powerful tool for biochemical analysis in general and in proteomics in particular. However, adaptation and taming of this molecular MS technique to the analysis of engineered NPs was not straightforward.

There are synergisms between NPs and MALDI-MS detection, which have been already widely exploited. In fact, some outstanding features of NPs can be exploited to improve the analytical features of the MALDI technique. For instance, discrete NPs can be used for the isolation/preconcentration of analytes prior to MALDI analysis [59]. Interestingly, NPs seem also to offer important advantages over more conventional organic MALDI matrices, due to the fact that some NPs are excellent laser-energy-absorbing materials, their comparatively high surface area, ease of MALDI sample preparation and flexibility of the sample deposition [60]. This, of course, facilitates analyte desorption and ionization processes, so enabling improvements of spectra acquisition, particularly in the low-mass range (e.g. amino acid, peptide, polymer and even metal analysis) where a lot 
of ions coming from the more conventional MALDI organic matrices are typically detected [61]. For example, $\mathrm{Fe}_{3} \mathrm{O}_{4} / \mathrm{TiO}_{2}$ core/shell NPs demonstrated a high selectivity toward phosphopeptides and were found to be advantageous matrices for the analysis of proteins, with up to 24,000 Da masses, by SALDI-MS [62].

Molecular MS has been also used for the identification of fragments derived from reagents adsorbed on the nanoparticle's surface. In this line, in a recent report, silver NPs with different surface capping reagents (able to interact with proteins and peptides such as insulin, heart cytochrome c, ubiquitin, lysozyme, cysteine, and homocysteine) were prepared [63]. Such functionalized AgNPs were applied in a single drop microextraction process. The proteins after separation were analyzed by MALDI-MS.

Additionally, MALDI-MS has been also investigated for characterization of functionalized NPs themselves. In such cases an appropriate matrix should be selected to minimize the formation of fragments from the NP itself (that might limit the possibility to assign unequivocally the correct molecular formula to the bioconjugate). In this sense, it was observed that the use of trans-2-[3-(4-tertbutylphenyl)-2-methyl-2-propenylidene]malononitrile (DCTB) as the MALDI matrix allows an efficient production of charged ions from the NPs [64]. Instead of the transfer of a proton, as typical MALDI matrices do, DCTB assists ionization of the sample through electron transfer properties and this induces less fragmentation.

In one of the first reports on NPs analysis by MALDI-MS, the technique was utilized to characterize platinum NPs, synthesized in the 1-4 nm size range, and capped with Poly(vinylpyrrolidone), PVP, in order to stabilize the PtNPs in solution [65]. A model for determining the size of the metallic nanoparticle core from MALDI-TOF mass spectra is proposed here and results were successfully verified by the correlation with particle sizes figures obtained by transmission electron microscopy and X-ray diffraction [65].

After those pioneering studies, extensive research on NPs characterization by MALDI has been reported [66-69]. Such MALDIMS investigations have demonstrated this MS-based technique capabilities for the characterization of a broad range of AuNPs (with cores ranging between 16-144 atoms of gold), and providing enough resolution to be able to discriminate between $\mathrm{Au}_{38}$ and $\mathrm{Au}_{40}$ clusters. However, there are still some remaining limitations, including robust determinations of unbroken higher mass NPs and of NPs protected with hydrophilic ligands.

The determination of NPs stoichiometry in bioconjugates and/ or the quantification of ligand mixtures that protect the metal core are currently important aspects of present research in this field. In this context, MALDI-Ion Mobility-MS [70] allowed to measure the relative quantity of ligands on a given AuNPs surface. The results were successfully validated by nuclear magnetic resonance (NMR) spectroscopy and by MS without ion mobility separation (in fact, average differences of less than $1 \%$ relative abundance were reported). In another recent example, the size and distribution of patuletin coated gold NPs have been established with MALDI-TOFMS [71]. Data obtained from the MALDI TOF-MS analysis of the NPs allowed to confirm the functionalization of the NPs with the patuletin and allowed to deduce that the ligand was bound to the gold surface through two phenol groups.

\subsection{Electrospray ionization}

Electrospray ionization (ESI) is an alternative soft ionization technique that might overcome the susceptibility of some molecules to fragment themselves when ionized from the solid matrix (e.g. MALDI). ESI constitutes a very popular today ionization source for liquid samples. Another advantage of ESI over MALDI is the production of multiply charged ions, effectively extending the mass range of the analyzer to accommodate KDa-MDa masses (extremely important in protein analysis). In this context, information about the charge states of NPs-based compounds can be also obtained by ESI-MS [72].

The usefulness of ESI-MS for characterization and optimization of novel NPs synthesis has been demonstrated by some authors. As an illustrative example, it was possible to improve the synthesis of gold nanoclusters and also establishing the composition of the $29 \mathrm{kDa}$ species, as Au144(SR)59, on the basis of detailed ESI-MS analysis [73].

Of course, in many cases ESI-MS and MALDI-MS offer complementary information of greater value for NPs characterization. As an example, both techniques have been simultaneously used during the characterization of the products resulting from the synthesis of $\mathrm{Au}_{102}(p-\mathrm{MBA})_{44} \mathrm{NPs}$ [69]. Information about molecular weight (22 $\mathrm{kDa}$ ) is given by MALDI-MS whereas ESI-MS is used to determine the chemical formulas and charge states of thiolate-protected gold clusters. Both types of results agreed and allowed the elucidation of the final molecular formula of the coated clusters of gold.

ESI-MS has also demonstrated an extraordinary potential and capabilities to analyse more complex samples containing NPs. In particular, samples containing bimetallic protected NPs (e.g. Au$\mathrm{Ag}, \mathrm{Pd}-\mathrm{Ag}, \mathrm{Au}-\mathrm{Pd}$, and $\mathrm{Cu}-\mathrm{Au}$ core-shell NPs) have been analyzed and the reaction products from ligand exchange reactions (e.g. $\mathrm{Au}_{25-\mathrm{x}} \mathrm{Pd}_{\mathrm{x}}\left(\mathrm{SC}_{2} \mathrm{Ph}\right)_{18-\mathrm{y}}(\mathrm{SPEG})_{\mathrm{y}}$, being SPEG=-S( $\left.\left.\mathrm{CH}_{2} \mathrm{CH}_{2} \mathrm{O}\right)_{5} \mathrm{CH}_{3}\right)$, could be monitored [74]: for Au/Pd bimetallic NPs, a more rapid ligand exchange during PEGylation was observed. In addition, ESI-MS has been also used as reference technique to validate results obtained by alternative new analytical tools. As an illustration of such application, the pioneering work on "Diffusion Ordered NMR spectroscopy" for size determination of thiol-stabilized gold NPs describes ESI-MS spectra obtained to confirm the results obtained by the NMR method [75].

\section{Conclusions and outlook on the future of mass spectrometry for engineered nanoparticles}

Nanoparticles have been extensively used as labels for optical imaging and sensing in bioscience [76]. However, in the last decades research efforts have been focused in the integration of the available MS-techniques to obtain both elemental and molecular information from engineered NPs, in the design of new compatible MS instrumentation and in the investigation of novel MS modes and processes to facilitate a more reliable and complete characterization of inorganic-based nanomaterials. By now MS-based techniques can be considered essential tools for the reliable characterization of NPs and so to apply and control their labeling exceptional features in the field.

To start with, elemental MS today does not only allow the determination of the multielemental (isotopic) NPs content, but also provides absolute quantitative information of great value for NP mass and number concentrations evaluation. Core/ligand ratios and/or determination of elemental stoichiometry within the desired NP can be established. In parallel, molecular MS techniques provide great promise for ligand coating and bioconjugation assessment of NP-based labelling reagents but have been scarcely used so far. Very importantly, a judicious combination of elemental and molecular MS-based techniques enable today to obtain very useful multiparametric information of the desired NP, including NP size, composition, stoichiometry, size distribution, number and mass elemental concentration, coverage density of ligands, synthesis and reaction processes monitoring, and, of course, eventual elucidation of molecular formula. Nevertheless, further improvements in both, instrumentation and methodologies, are still mandatory to achieve higher sensitivities, better resolution and more accurate and reproducible analytical results. 
Methods to efficiently determine the size, polydispersity, and concentrations of nanomaterials in liquid suspensions are extremely important in nanomanufacturing systems, particularly for nanomaterials (nanoparticles) in the low nm size range. Recently, IMS specifically using a differential mobility analyzer (DMA), a technique typically developed for aerosol analysis, has been also investigated for the analysis of liquid nanoparticle suspensions, provided that the particles of interest can be aerosolized preserving their size distribution function [77]. Though this technique has been successfully applied to metal nanoparticles there are still drawbacks to using electrosprays for aerosolization (e.g. the rather strict requirements on the electrical conductivities of the dispersions to be electrosprayed and the fact that non-volatile solutes need to be removed from the dispersion prior to electrospray based aerosolization). Thus, IMS approaches have not been widely adopted yet for NPs size distribution analysis in liquids, even if recent trends in this topic include the development of novel improved liquid nebulizers to aerosolize NPs. Some improvements, demonstrated recently [78], enable the measurements of size distribution of gold nanospheres in the 5-30 nm range. Still, further refinement of this promising technique will be necessary for improved analytical accuracy.

The growing role of MS-based hyphenated techniques to detect, quantify and characterize different NPs (and so boost their importance and impact in varied application fields) is undebatable today. The combination of a powerful NP separation technique with the outstanding features of MS-based detection are gaining momentum in the active research field of novel functionalized nanoparticle characterization. A particular field of promising future of such hybrid technique is the investigations on the protein adsorption layer (named the "protein corona") that forms on the surface of NPs when they interact with biological fluids and tissues. Characterization of the "protein corona" is of utmost importance these days for a better understanding of how exposure to NPs affects the biological responses of cells and organisms. Although quite a lot of experimental studies have been reported in this direction, a comprehensive picture is still missing [79]. The direct determination of the proteins attached per NP is not straightforward. It is difficult not only to quantify the amount of attached proteins, but also to identify and specify the individual proteins forming the protein corona. A classical method for such characterizations is enzymatic digestion of the proteins bound to the NP surface, and their subsequent separation by polyacrylamide gel electrophoresis with different identification and/or quantification strategies (e.g. by MS).

Among the different approaches, a recent trend in the area, is the use of more general "omics" tools. Such approaches have been recently proposed to study NP bioactivity and interaction with cells at a molecular level. In particular, ZnONPs, $\mathrm{TiO}_{2} \mathrm{NPs}$, NiONPs, PtNPs and AgNPs have been studied, by means of soft ionization MS techniques (e.g. ESI- and MALDI-MS) [80]. The study focuses on studying the interactions of five different unmodified (bare) nanoparticles based on their interactions with two bacteria and proposed the interaction-mechanism based on the results obtained from MALDIMS, UV-vis and TEM studies. Particularly, weakening the cell membranes and the interaction of the NPs with the internal proteins enables more signals during MALDI-MS analysis. This effect has been used to study the NP-cell interaction.

Methods based on MS have been reported also for a timedependent analysis of the composition of the protein corona [81]. However, so far the systems are too complex to allow reliable extraction of quantitative parameters of the individual protein species of the "corona". Future advances in characterization methodologies (e.g. using separation techniques, such as AF4, with elemental and molecular MS) are required in order to lead to get new insights and a better understanding of the formation and composition of the protein corona.
No doubt, recent developments on sp-ICP-MS operation mode constitute an important breakthrough aiming at a direct NPs analysis. The success of sp-ICP-MS lies on the fact that several and different types of analytical information can be obtained directly from the sample (e.g. qualitative information about the presence of particulate and/or dissolved forms, quantitative information on particle number concentrations and of course, information about the mass of element/s per particle and the corresponding particle size).

One important aspect in sp-ICP-MS relies on its capability to differentiate engineered (artificial) and natural nanoparticles in environmental science. This is clearly a complex problem in environmental issues due to the ubiquitous presence of different types of natural NPs (covering a broad range of sizes) along with the increasing levels and varieties of engineered NPs posing a risk to our health.

In order to distinguish engineered NPs from natural NPs of similar composition, some approaches make use of the fact that natural NPs have metallic cores with different elemental or isotopic ratios from those observed in the engineered NPs. In this vein, the monitoring of elemental ratios in individual particles by sp-ICP-MS allowed the correct identification of engineered and natural NPs in a particleby-particle basis [82].

Although a lot of work has already been done so far, further efforts are still needed to enable the robust analysis of smaller metal NPs [83] approaching typical NP-sizes (around 1-20 nm) used in bioanalytical applications. In addition, the development of novel mass spectrometers able to monitor pulses of fast transient signals (as those originated normally by atomization of a single particle in a plasma) with enough time resolution, represents a current unresolved challenge. It is expected that continuous ongoing developments of the ICP-MS instrumentation will allow to overcome such hurdles in the near future.

Last but not least, further advances in applying molecular MS (MALDI and ESI) for the molecular characterization of NPs and their bioconjugates in general should be directed to minimize the fragmentation of the ligands/NPs association in order to increase the certainty of molecular formula assignments. In this vein, improving sample quality, mass resolving power, and mass measurement accuracy are urgently demanded to minimize nearlyisobaric permutations presently observed. Particularly "nanoparticle handling and sample preparation" is a pending challenge as it would strongly depend on the nanoparticle type, on the coating and on the nature (matrix) of the analyzed sample. The preparation of stable NP dispersions, as reliable standards, is probably one of the more urgent issues to be addressed, especially for size-fractionated NPs analysis.

The ongoing MS developments will facilitate the controlled synthesis and production of NP-based elemental labels. Moreover, such developments will provide invaluable information about the myriad of synthesized novel NP bioconjugates.

\section{Acknowledgments}

Financial support from the Ministry of Economy and Competitiveness (MINECO-13-CTQ2013-49032) and from Gobierno del Principado de Asturias (FC-15-GRUPIN14-092) is gratefully acknowledged. Diego Bouzas-Ramos and Mario Menéndez-Miranda acknowledge their Ph.D. grants (BP14-137 and BP12-046, respectively) from Principado de Asturias (Spain).

\section{References}

[1] International Organization for Standardization (ISO), ISO TS27687; ISO, Geneva, Switzerland, 2008. 
[2] S.J. Soenen, P. Rivera-Gil, J.-M. Montenegro, W.J. Parak, S.C. De Smedt, K. Braeckmans, Cellular toxicity of inorganic nanoparticles: common aspects and guidelines for improved nanotoxicity evaluation, Nano Today 6 (2011) 446-465.

[3] A.I. López-Lorente, M. Valcárcel, The third way in analytical nanoscience and nanotechnology: involvement of nanotools and nanoanalytes in the same analytical process, Trends Anal. Chem. 75 (2016) 1-9.

[4] A.R. Montoro-Bustos, J.R. Encinar, A. Sanz-Medel, Mass spectrometry for the characterisation of nanoparticles, Anal. Bioanal. Chem. 405 (2013) 5637-5643.

[5] B. Fernández, J.M. Costa, R. Pereiro, A. Sanz-Medel, Inorganic mass spectrometry as a tool for characterisation at the nanoscale, Anal. Bioanal. Chem. 396 (2010) 15-29.

[6] R. Allabashi, W. Stach, A. de la Escosura-Muñiz, L. Liste-Calleja, A. Merkoçi, ICP-MS: a powerful technique for quantitative determination of gold nanoparticles without previous dissolving, J. Nanopart. Res. 11 (2009) 20032011.

[7] L. Duester, D. Rakcheev, J.V. Bayer, P.M. Abraham, A. Dabrunz, R. Schulz, et al., A robust, particle size independent, method for quantifying metal(loid oxide) nanoparticles and their agglomerates in complex environmental matrices by electrothermal vaporisation coupled to ICP-MS, J. Anal. At. Spectrom. 26 (2011) $450-455$.

[8] X. Jiang, K. Huang, D. Deng, H. Xia, X. Hou, C. Zheng, Nanomaterials in analytical atomic spectrometry, Trends Anal. Chem. 39 (2012) 38-59.

[9] X. He, H. Xie, X. Nie, Y. Tang, Determination of trace impurities in high purity titanium dioxide by high resolution inductively coupled plasma mass spectrometry, Spectrosc. Spectr. Anal. 27 (2007) 1192-1196.

[10] I. Moreels, K. Lambert, D. De Muynck, F. Vanhaecke, D. Poelman, J.C. Martins, et al., Composition and size-dependent extinction coefficient of colloidal $\mathrm{PbSe}$ quantum dots, Chem. Mater. 19 (2007) 6101-6106.

[11] J.H. Bang, W.H. Suh, K.S. Suslick, Quantum dots from chemical aerosol flow synthesis: preparation, characterization, and cellular imaging, Chem. Mater. 20 (2008) 4033-4038.

[12] A.R.M. Bustos, J.R. Encinar, M.T. Fernández-Argüelles, J.M. Costa-Fernández, A. Sanz-Medel, Elemental mass spectrometry: a powerful tool for an accurate characterisation at elemental level of quantum dots, Chem. Commun. (Camb.) (2009) 3107-3109.

[13] D. Bouzas-Ramos, M. Menéndez-Miranda, J.M. Costa-Fernández, J.R. Encinar, A. Sanz-Medel, Precise determination of the nanoparticle concentration and ligand density of engineered water-soluble HgSe fluorescent nanoparticles, RSC Adv. 6 (2016) 19964-19972.

[14] W. Jiang, D.B. Hibbert, G. Moran, R. Akter, Measurement of gold and sulfur mass fractions in L-cysteine-modified gold nanoparticles by ICP-DRC-MS after acid digestion: validation and uncertainty of results, J. Anal. At. Spectrom. 27 (2012) $1465-1473$

[15] H. Hinterwirth, S. Kappel, T. Waitz, T. Prohaska, W. Lindner, M. Lämmerhofer, Quantifying thiol ligand density of self-assembled monolayers on gold nanoparticles by inductively coupled plasma-mass spectrometry, ACS Nano 7 (2013) 1129-1136.

[16] L. Trapiella-Alfonso, J.M. Costa-Fernández, J.R. Encinar, R. Pereiro, A. Sanz-Medel, Mass spectrometry for the characterization of gold nanoparticles, Compr. Anal. Chem. 66 (2014) 329-356. In: M. Valcárcel, A.I. López-Lorente (Editors), Gold nanoparticles in analytical chemistry

[17] J. Tang, L. Xiong, S. Wang, J. Wang, L. Liu, J. Li, et al., Influence of silver nanoparticles on neurons and blood-brain barrier via subcutaneous injection in rats, Appl. Surf. Sci. 255 (2008) 502-504.

[18] Z. Chen, H. Chen, H. Meng, G. Xing, X. Gao, B. Sun, et al., Bio-distribution and metabolic paths of silica coated CdSeS quantum dots, Toxicol. Appl. Pharmacol. 230 (2008) 364-371.

[19] E. Caballero-Díaz, C. Pfeiffer, L. Kastl, P. Rivera-Gil, B. Simonet, M. Valcárcel, et al., The toxicity of silver nanoparticles depends on their uptake by cells and thus on their surface chemistry, Part. Part. Syst. Charact. 30 (2013) 10791085.

[20] R.S.H. Yang, L.W. Chang, J. Wu, M. Tsai, H. Wang, Y. Kuo, et al., Persistent tissue kinetics and redistribution of nanoparticles, quantum dot 705, in mice: ICP-MS quantitative assessment, Environ. Health Perspect. 115 (2007) 1339-1343.

[21] A.R. Timerbaev, Role of mass spectrometry in the development and medicinal implementation of metal-based nanoparticles, J. Anal. Chem. 70 (2015) 1031-1046.

[22] C. Degueldre, P.Y. Favarger, Colloid analysis by single particle inductively coupled plasma-mass spectroscopy: a feasibility study, Colloids Surf. A Physicochem. Eng. Asp. 217 (2003) 137-142.

[23] F. Laborda, E. Bolea, J. Jiménez-Lamana, Single particle inductively coupled plasma mass spectrometry: a powerful tool for nanoanalysis, Anal. Chem. 86 (2014) 2270-2278.

[24] S.M. Majedi, H.K. Lee, Recent advances in the separation and quantification of metallic nanoparticles and ions in the environment, Trends Anal. Chem. 75 (2016) 183-196.

[25] R.B. Reed, C.P. Higgins, P. Westerhoff, S. Tadjiki, J.F. Ranville, Overcoming challenges in analysis of polydisperse metal-containing nanoparticles by single particle inductively coupled plasma mass spectrometry, J. Anal. At. Spectrom. 27 (2012) 1093-1100.

[26] F. Laborda, E. Bolea, G. Cepriá, M.T. Gómez, M.S. Jiménez, J. Pérez-Arantegui, et al., Detection, characterization and quantification of inorganic engineered nanomaterials: a review of techniques and methodological approaches for the analysis of complex samples, Anal. Chim. Acta 904 (2016) 10-32.

[27] O. Borovinskaya, S. Gschwind, B. Hattendorf, M. Tanner, D. Günther, Simultaneous mass quantification of nanoparticles of different composition in a mixture by microdroplet generator-ICPTOFMS, Anal. Chem. 86 (2014) $8142-8148$

[28] O.A. Sadik, N. Du, V. Kariuki, V. Okello, V. Bushlyar, Current and emerging technologies for the characterization of nanomaterials, ACS Sustain. Chem. Eng. 2 (2014) 1707-1716.

[29] A.R. Montoro-Bustos, E.J. Petersen, A. Possolo, M.R. Winchester, Post hoc interlaboratory comparison of single particle ICP-MS size measurements of NIST gold nanoparticle reference materials, Anal. Chem. 87 (2015) 8809-8817.

[30] G. Schmid, Nanoparticles: From Theory to Application, Wiley-VCH, Weinheim, 2003.

[31] K.M. Krueger, A.M. Al-Somali, J.C. Falkner, V.L. Colvin, Characterization of nanocrystalline CdSe by size exclusion chromatography, Anal. Chem. 77 (2005) 3511-3515

[32] A.M. Al-Somali, K.M. Krueger, J.C. Falkner, V.L. Colvin, Recycling size exclusion chromatography for the analysis and separation of nanocrystalline gold, Anal. Chem. 76 (2004) 5903-5910.

[33] G.S. Duesberg, J. Muster, V. Krstic, M. Burghard, S. Roth, Chromatographic size separation of single-wall carbon nanotubes, Appl. Phys. A Mater. Sci. Process. 67 (1998) 117-119.

[34] L. Trapiella-Alfonso, A.R. Montoro, J.R. Encinar, J.M. Costa-Fernandez, R. Pereiro, A. Sanz-Medel, New integrated elemental and molecular strategies as a diagnostic tool for the quality of water soluble quantum dots and their bioconjugates, Nanoscale 3 (2011) 954-957.

[35] P. Paydary, P. Larese-Casanova, Separation and quantification of quantum dots and dissolved metal cations by size exclusion chromatography-ICP-MS, Int. J. Environ. Anal. Chem. 95 (2015) 1450-1470.

[36] W.G. Kreyling, A.M. Abdelmonem, Z. Ali, F. Alves, M. Geiser, N. Haberl, et al., In vivo integrity of polymer-coated gold nanoparticles, Nat. Nanotechnol. 10 (2015) 619-623.

[37] G.T. Wei, F.K. Liu, C.R.C. Wang, Shape separation of nanometer gold particles by size-exclusion chromatography, Anal. Chem. 71 (1999) 2085-2091.

[38] K. Tiede, A.B.A. Boxall, D. Tiede, S.P. Tear, H. David, J. Lewis, A robust sizecharacterisation methodology for studying nanoparticle behaviour in 'real' environmental samples, using hydrodynamic chromatography coupled to ICP-MS, J. Anal. At. Spectrom. 24 (2009) 964-972.

[39] S.A. Pergantis, T.L. Jones-Lepp, E.M. Heithmar, Hydrodynamic chromatography online with single particle-inductively coupled plasma mass spectrometry for ultratrace detection of metal-containing nanoparticles, Anal. Chem. 84 (2012) 6454-6462.

[40] M. Roman, C. Rigo, H. Castillo-Michel, I. Munivrana, V. Vindigni, I. Mičetić, et al., Hydrodynamic chromatography coupled to single-particle ICP-MS for the simultaneous characterization of AgNPs and determination of dissolved $\mathrm{Ag}$ in plasma and blood of burn patients, Anal. Bioanal. Chem. (2015) doi:10.1007/ s00216-015-9014-6.

[41] B. Roda, A. Zattoni, P. Reschiglian, M.H. Moon, M. Mirasoli, E. Michelini, et al., Field-flow fractionation in bioanalysis: a review of recent trends, Anal. Chim. Acta 635 (2009) 132-143.

[42] J. Gigault, J.M. Pettibone, C. Schmitt, V.A. Hackley, Rational strategy for characterization of nanoscale particles by asymmetric-flow field flow fractionation: a tutorial, Anal. Chim. Acta 809 (2014) 9-24.

[43] T. Rameshwar, S. Samal, S. Lee, S. Kim, J. Cho, I.S. Kim, Determination of the Size of Water-Soluble Nanoparticles and Quantum Dots by Field-Flow Fractionation, J. Nanosci. Nanotechnol. 6 (2006) 2461-2467.

[44] C. Contado, A. Pagnoni, TiO2 in commercial sunscreen lotion: flow field-flow fractionation and ICP-AES together for size analysis, Anal. Chem. 80 (2008) 7594-7608.

[45] M. Bouby, H. Geckeis, F.W. Geyer, Application of asymmetric flow field-flow fractionation (AsFlFFF) coupled to inductively coupled plasma mass spectrometry (ICPMS) to the quantitative characterization of natural colloids and synthetic nanoparticles, Anal. Bioanal. Chem. 392 (2008) 14471457.

[46] M. Menendez-Miranda, M.T. Fernandez-Arguelles, J.M. Costa-Fernandez, J. Ruiz, A. Sanz-Medel, Elemental ratios for characterization of quantum-dots populations in complex mixtures by asymmetrical flow field-flow fractionation online coupled to fluorescence and inductively coupled plasma mass spectrometry, Anal. Chim. Acta 839 (2014) 8-13.

[47] M. Menendez-Miranda, J. Ruiz, J.M. Costa-Fernández, A. Sanz-Medel, Asymmetric flow field-flow fractionation coupled to inductively coupled plasma mass spectrometry for the quantification of quantum dots bioconjugation efficiency, J. Chromatogr. A 1422 (2015) 247-252.

[48] S. Carter, A. Fisher, R. Garcia, B. Gibson, S. Lancaster, J. Marshallf, et al., Atomic spectrometry update. Review of advances in the analysis of metals, chemicals and functional materials, J. Anal. At. Spectrom. 30 (2015) 2249-2294.

[49] J. Gigault, V.A. Hackley, Differentiation and characterization of isotopically modified silver nanoparticles in aqueous media using asymmetric-flow field flow fractionation coupled to optical detection and mass spectrometry, Anal. Chim. Acta 763 (2013) 57-66.

[50] D.M. Mitrano, A. Barber, A. Bednar, P. Westerhoff, C.P. Higgins, J.F. Ranville, Silver nanoparticle characterization using single particle ICP-MS (sp-ICP-MS) and asymmetrical flow field flow fractionation ICP-MS (AF4-ICP-MS), J. Anal. At. Spectrom. 27 (2012) 1131-1142.

[51] A.B. Kanu, P. Dwivedi, M. Tam, L. Matz, H.H. Hill Jr., Ion mobility-mass spectrometry, J. Mass Spectrom. 43 (2008) 1-22.

[52] E.A. Kapellios, S.A. Pergantis, Size and elemental composition of nanoparticles using ion mobility spectrometry with inductively coupled plasma mass spectrometry, J. Anal. At. Spectrom. 27 (2012) 21-24. 
[53] S. Elzey, T. De-Ha, L.L. Yu, M.R. Winchester, M.E. Kelley, V.A. Hackley, Real-time size discrimination and elemental analysis of gold nanoparticles using ES-DMA coupled to ICP-MS, Anal. Bioanal. Chem. 405 (2013) 2279-2288.

[54] A.I. López-Lorente, B.M. Simonet, M. Valcárcel, Electrophoretic methods for the analysis of nanoparticles, Trends Anal. Chem. 30 (2011) 58-71.

[55] M.S. da Rocha, A.B. Soldado, E. Blanco-Gonzalez, A. Sanz-Medel, Speciation of mercury compounds by capillary electrophoresis coupled on-line with quadrupole and double-focusing inductively coupled plasma mass spectrometry, J. Anal. At. Spectrom. 15 (2000) 513-518.

[56] L. Liu, B. He, Q. Liu, Z. Yun, X. Yan, Y. Long, et al., Identification and accurate size characterization of nanoparticles in complex media, Angew. Chem. Int. Ed. Engl. 53 (2014) 14476-14479.

[57] H. Qu, T.K. Mudalige, S.W. Linder, Capillary electrophoresis/inductively coupled plasma-mass spectrometry: development and optimization of a high resolution analytical tool for the size-based characterization of nanomaterials in dietary supplements, Anal. Chem. 86 (2014) 11620-11627.

[58] T. Jamieson, R. Bakhshi, D. Petrova, R. Pocock, M. Imani, A.M. Seifalian, Biological applications of quantum dots, Biomaterials 28 (2007) 4717.

[59] K. Turney, T.J. Drake, J.E. Smith, W. Tan, W.W. Harrison, Functionalized nanoparticles for liquid atmospheric pressure matrix-assisted laser desorption/ ionization peptide analysis, Rapid Commun. Mass Spectrom. 18 (2004) 23672374.

[60] Z. Guo, A.A.A. Ganawi, Q. Liu, L. He, Nanomaterials in mass spectrometry ionization and prospects for biological application, Anal. Bioanal. Chem. 384 (2006) 584-592.

[61] R. Pilolli, N. Ditaranto, C. Di Franco, F. Palmisano, N. Cioffi, Thermally annealed gold nanoparticles for surface-assisted laser desorption ionisation-mass spectrometry of low molecular weight analytes, Anal. Bioanal. Chem. 404 (2012) 1703-1711.

[62] C.-T. Chen, Y.-C. Chen, $\mathrm{Fe}_{3} \mathrm{O}_{4} / \mathrm{TiO}_{2}$ Core/Shell nanoparticles as affinity probes for the analysis of phosphopeptides using $\mathrm{TiO}_{2}$ surface-assisted laser desorption/ ionization mass spectrometry, Anal. Chem. 77 (2005) 5912-5919.

[63] L. Shastri, H.N. Abdelhamid, M. Nawaz, H.-F. Wu, Synthesis, characterization and bifunctional applications of bidentate silver nanoparticle assisted single drop microextraction as a highly sensitive preconcentrating probe for protein analysis, RSC Adv. 5 (2015) 41595-41603.

[64] A. Dass, A. Stevenson, G.R. Dubay, J.B. Tracy, R.W. Murray, Nanoparticle MALDI-TOF mass spectrometry without fragmentation: $\mathrm{Au}_{25}\left(\mathrm{SCH}_{2} \mathrm{CH}_{2} \mathrm{Ph}\right)_{18}$ and mixed monolayer $\mathrm{Au}_{25}\left(\mathrm{SCH}_{2} \mathrm{CH}_{2} \mathrm{Ph}\right)_{18}-\mathrm{x}(\mathrm{L}) \mathrm{x}, \mathrm{J}$. Am. Chem. Soc. 130 (2008) 5940-5946.

[65] J.K. Navin, M.E. Grass, G.A. Somorjai, A.L. Marsh, Characterization of colloidal platinum nanoparticles by MALDI-TOF mass spectrometry, Anal. Chem. 81 (2009) 6295-6299.

[66] S.M. Reilly, T. Krick, A. Dass, Surfactant-free synthesis of ultrasmall gold nanoclusters, J. Phys. Chem. C 114 (2010) 741-745.

[67] H. Qian, Y. Zhu, R. Jin, Isolation of ubiquitous Au40(SR)24 clusters from the $8 \mathrm{kDa}$ gold clusters, J. Am. Chem. Soc. 132 (2010) 4583-4585.
[68] P.R. Nimmala, A. Dass, Au36(SPh)23 nanomolecules, J. Am. Chem. Soc. 133 (2011) 9175-9177.

[69] Y. Levi-Kalisman, P.D. Jadzinsky, N. Kalisman, H. Tsunoyama, T. Tsukuda, D.A. Bushnell, et al., Synthesis and characterization of Au102(p-MBA)44 nanoparticles, J. Am. Chem. Soc. 133 (2011) 2976-2982.

[70] K.M. Harkness, B.C. Hixson, L.S. Fenn, B.N. Turner, A.C. Rape, C.A. Simpson, et al., A structural mass spectrometry strategy for the relative quantitation of ligands on mixed monolayer-protected gold nanoparticles, Anal. Chem. 82 (2010) 9268-9274.

[71] M. Ateeq, M.R. Shah, N. Ain, S. Bano, I. Anis, L. Ubna, et al., Green synthesis and molecular recognition ability of patuletin coated gold nanoparticles, Biosens. Bioelectron. 63 (2015) 499-505.

[72] J.J. Pitt, Principles and applications of liquid chromatography-mass spectrometry in clinical biochemistry, Clin. Biochem. Rev. 30 (2009) 19-34.

[73] H. Tsunoyama, Y. Negishi, T. Tsukuda, Chromatographic isolation of "missing" Au55 clusters protected by alkanethiolates, J. Am. Chem. Soc. 128 (2006) 6036-6037.

[74] C.A. Fields-Zinna, M.C. Crowe, A. Dass, J.E.F. Weaver, R.W. Murray, Mass spectrometry of small bimetal monolayer-protected clusters, Langmuir 25 (2009) 7704-7710.

[75] K. Salorinne, T. Lahtinen, J. Koivisto, E. Kalenius, M. Nissinen, M. Pettersson, et al, Nondestructive size determination of thiol-stabilized gold nanoclusters in solution by diffusion ordered NMR spectroscopy, Anal. Chem. 85 (2013) 3489-3492.

[76] A.M. Coto, E. Sotelo, M.T. Fernandez-Argüelles, R. Pereiro, J.M. Costa-Fernandez, A. Sanz-Medel, Nanoparticles as fluorescent labels for optical imaging and sensing in genomics and proteomics, Anal. Bioanal. Chem. 399 (2011) 29-42.

[77] S. Elzey, D.H. Tsai, L.L. Yu, M.R. Winchester, M.E. Kelley, V.A. Hackley, Real-time size discrimination and elemental analysis of gold nanoparticles using ES-DMA coupled to ICP-MS, Anal. Bioanal. Chem. 405 (2013) 2279-2288.

[78] S. Jeon, D.R. Oberreit, G.V. Schooneveld, C.J. Hogan Jr., Nanomaterial size distribution analysis via liquid nebulization coupled with ion mobility spectrometry (LN-IMS), Analyst 141 (2016) 1363-1375.

[79] P. del Pino, B. Pelaz, Q. Zhang, P. Maffre, G.U. Nienhausb, W.J. Parak, Protein corona formation around nanoparticles - from the past to the future, Mater. Horiz. 1 (2014) 301-313.

[80] J. Gopal, M. Manikandan, N. Hasan, C.H. Lee, H.F. Wu, A comparative study on the mode of interaction of different nanoparticles during MALDI-MS of bacterial cells, J. Mass Spectrom. 48 (2013) 119-127.

[81] S. Tenzer, D. Docter, S. Rosfa, A. Wlodarski, J. Kuharev, A. Rekik, et al., ACS Nano 5 (2011) 7155-7167.

[82] M.D. Montaño, G.V. Lowry, F. von der Kammer, J. Blue, J.F. Ranville, Current status and future direction for examining engineered nanoparticles in natural systems, Environ. Chem. 11 (2014) 351-366.

[83] S. Lee, X. Bi, R.B. Reed, J.F. Ranville, P. Herckes, P. Westerhoff, Nanoparticle size detection limits by single particle ICP-MS for 40 elements, Environ. Sci. Technol. 48 (2014) 10291-10300. 